\title{
1. The concept of nation
}

Whether one is considering international trade or the international monetary system, one cannot develop a rigorous analysis unless making clear the meaning of the terms used, in this case the word 'international'. But speaking of an international phenomenon necessarily leads to questioning the notion of 'nation' (since international means 'between nations'), as international exchanges are exchanges between traders located in different nations, and the international monetary system is a system which involves several nations. Thus, a meaning cannot be given to the word 'international' without having first defined a nation.

Looking for an economic definition of the nation is an old problem, which first concerned trade. In fact, for a theory of international trade to be developed, there must be differences between international trade and intra-national trade. Of course, in both cases, the same general theory is used, that is the general theory of exchange (which is covered in Chapter 2). But the theory of international trade has to add a specification, so that it can be a particular application of the general theory of exchange.

One of the criteria that has long been used and which is still used most frequently in the theory of international trade is the criterion of the mobility of goods and factors of production. Most generally, economic theory assumes that goods and factors of production are perfectly mobile, which means that they can move without costs. One may study the consequences of a restriction on mobility (for example, arising from transport costs or information costs). Traditionally the trade theory assumes - as hypothesized by David Ricardo - that commodities are internationally mobile, whereas factors of production are mobile within a nation, but immobile internationally. In other words, a nation in the economic-commercial sense can be defined as the space within which factors of production are perfectly mobile, although they are totally immobile between nations. There is no need at this stage to investigate the specific causes of this immobility (natural or linguistic barriers, state controls and bans on trade, and so on).

This traditional definition therefore contrasted 'perfect' mobility for commodities and factors of production within the nation, to the 'total' immobility of factors of production between nations. But one can give a 
less extreme version of this theory by defining a nation according to the assumption that the mobility of factors of production is relatively greater within a nation than between nations. Thus, the mobility of factors is not necessarily 'perfect' ${ }^{1}$ - without cost - inside the nation, and not necessarily non-existent between nations. Moreover the theory of protection is precisely designed to analyse what is happening when the international mobility of commodities is not 'perfect' (tariffs, quotas or state authorizations constituting barriers to exchange).

Thus, traditionally, international economics retains a definition of the nation which could be called a commercial definition, since it is concerned with trade in commodities. But there can obviously be all sorts of other definitions of the nation using other criteria. Thus, we could define a nation in the linguistic sense as the space in which a language is mobile in absolute or relative terms; that is, the language in question is the most commonly used communication tool between residents of this space, while it is relatively less used in their dealings with residents of other linguistic areas. One could even, of course, define a nation in the gastronomic sense (circulation in a gastronomic space) or the artistic sense. But most often it is the legal and institutional meaning which is considered in defining a nation. From this point of view, a nation is the place in which a given system of law is used (or is 'mobile', while it is not between different nations). However, nowadays we usually consider that the creation of law is made by a state monopoly, contrary to what exists in jurisprudential systems in which the law is revealed by the judges, on the basis of the general principles of law (which can be considered as universal and not strictly 'national'). Thus, in present systems, there is a coincidence between the legal space and the political space (in which the state exercises its power). But, from a more general point of view, there is not necessarily such a coincidence between the political and the legal space.

This coincidence, so common in our time, does not correspond to any 'natural' need. It is the consequence of the working of the political power, which gets a monopoly of legal coercion on a space called the 'nation' and which uses its power of coercion to impose its monopoly in the development and the implementation of legal rules (laws and regulations).

What will mainly be used in this book, however, is the monetary

1 The use of the term 'perfect' is traditional, but it can lead to some ambiguity. When it is said that mobility is 'perfect', it is not to state a normative proposal representing an ideal which should be moved towards. Indeed, the reality is necessarily 'imperfect' in the sense that there are necessarily obstacles to trade, in particular the fact that information can never be 'perfect'. But the assumption of perfection must be considered as an instrument to facilitate a first step in reasoning. 
definition of the nation. Using criteria similar to those given above, a nation can be defined in the monetary sense as a space within which the currency is perfectly 'mobile', while it is immobile between nations. Of course, a relative definition can also be provided of the 'monetary nation', described as a space within which the currency is relatively more mobile than it is between 'monetary nations'.

But what does the mobility of a currency mean? ${ }^{2}$ It is simply the fact that it is accepted as a medium of exchange in transactions. But, as we shall see, the acceptability of a currency may result from a selection process voluntarily implemented by those concerned, or the result of a legal obligation to use a given currency, in which case one speaks of 'legal tender'. Thus the use and circulation of currencies can be the result of the freely expressed choice of individuals or the result of an obligation imposed by states using their legal right to coerce. This is obviously a fundamental distinction, but it is unfortunately all too often neglected in analyses of monetary systems. The fact remains that with currencies the same problem arises as with the law: there is no reason a priori for the monetary space - namely, the space of circulation of a currency - to coincide with the political space. But nowadays the political power uses its power of coercion to impose a monopoly on the production and circulation of money, which in particular implies the existence of a 'legal tender', that is, a prohibition on currencies other than the 'national' currency.

When speaking of the present-day international monetary system, one makes the implicit assumption - which in fact is coherent with the modern reality - that all currencies have a 'national' character in the political sense, that is, that there is coincidence between the nation in the monetary sense and the nation in a political sense. The study of the international monetary system, then, consists in finding out the role of different national currencies in transactions which take place between people using different national currencies. But to understand the operation of an international monetary system' it seems important to adopt a broader perspective by first analysing relations between currencies belonging to different monetary areas in cases in which there is no coincidence between the monetary area and the political area. This being done, it is then possible to add the assumption that this coincidence exists - as is generally the case

2 What is meant by 'a currency' should also be defined. Indeed, a currency is composed of a set of more or less heterogeneous monetary instruments (for instance, banknotes and deposits, possibly produced by different organizations). What makes it possible to say that these instruments constitute a single currency? I will not give an answer to this question here, but will do so in detail later on. But this issue is at the heart of any analysis of monetary systems. 
nowadays - and to look for its implications. The analysis of the international monetary system is therefore only a particular application of a more general analysis, namely the theory of monetary systems. One will thus better understand the functioning of the international monetary system if one has first explored the broader operating processes which concern all monetary systems. But to avoid confusion, this general analysis of the monetary systems will not use the term 'nation' (although, as we have seen, a nation in the monetary sense can be defined), but will instead use the term 'monetary area' or 'monetary zone'. And use of the term 'nation' will be reserved for when there is coincidence between the monetary and political areas, which means that 'nation' refers to a reality of political order.

In other words, the theory of monetary systems is concerned with both the study of the internal structure of a monetary system and the study of relations between different monetary systems. Insofar as there is a plurality of currencies, and therefore a plurality of systems of production and circulation of currency, there are necessarily problems of relations between these different systems. But there are some specific characteristics in the functioning of these systems and of their interrelationships, depending on whether currencies are 'national' or not. 